\title{
16 CONCLUSION
}

Direct evidence of a role for molybdenum in substrate binding and of an involvement in the catalytic phase of enzyme activity, is available only for xanthine oxidase. The marked similarities between the e.p.r. parameters and substrate specificities of this enzyme and those of aldehyde oxidase suggest that the molybdenum is also present at the substrate binding site in the latter enzyme. Limited e.p.r. data also locate molybdenum at this site in nitrate reductase from $M$. denitrificans and in mammalian and avian sulphite oxidase. Studies of molybdenum complexes as models of the enzymes have centred on complexes with sulphur-containing ligands, especially cysteine, although there is only meagre evidence for sulphur-liganded molybdenum in the enzymes. A molybdenumcysteine complex exhibits reductase activity towards several substrates that are also substrates for nitrogenase but fails to produce more than trace yields of ammonia from molecular nitrogen. The models also differ appreciably from the enzymes in their content of e.p.r.-active $\mathrm{Mo}(\mathrm{V})$. This is as high as about 50 per cent in the reduced oxidase enzymes, but does not exceed more than about 4 per cent in the models. Possibly, the conformation of the protein at the active sites of the enzymes results in the stabilisation of catalytically active, monomeric molybdenum species. But this, like the various proposed schemes of nitrogenase action, remain speculative in the absence of structural data. 